\title{
Front Matter: Volume 8428
}

, "Front Matter: Volume 8428," Proc. SPIE 8428, Micro-Optics 2012, 842801 (18 May 2012); doi: 10.1117/12.940786

SPIE. Event: SPIE Photonics Europe, 2012, Brussels, Belgium 


\section{PROCEEDINGS OF SPIE}

\section{Micro-Optics 2012}

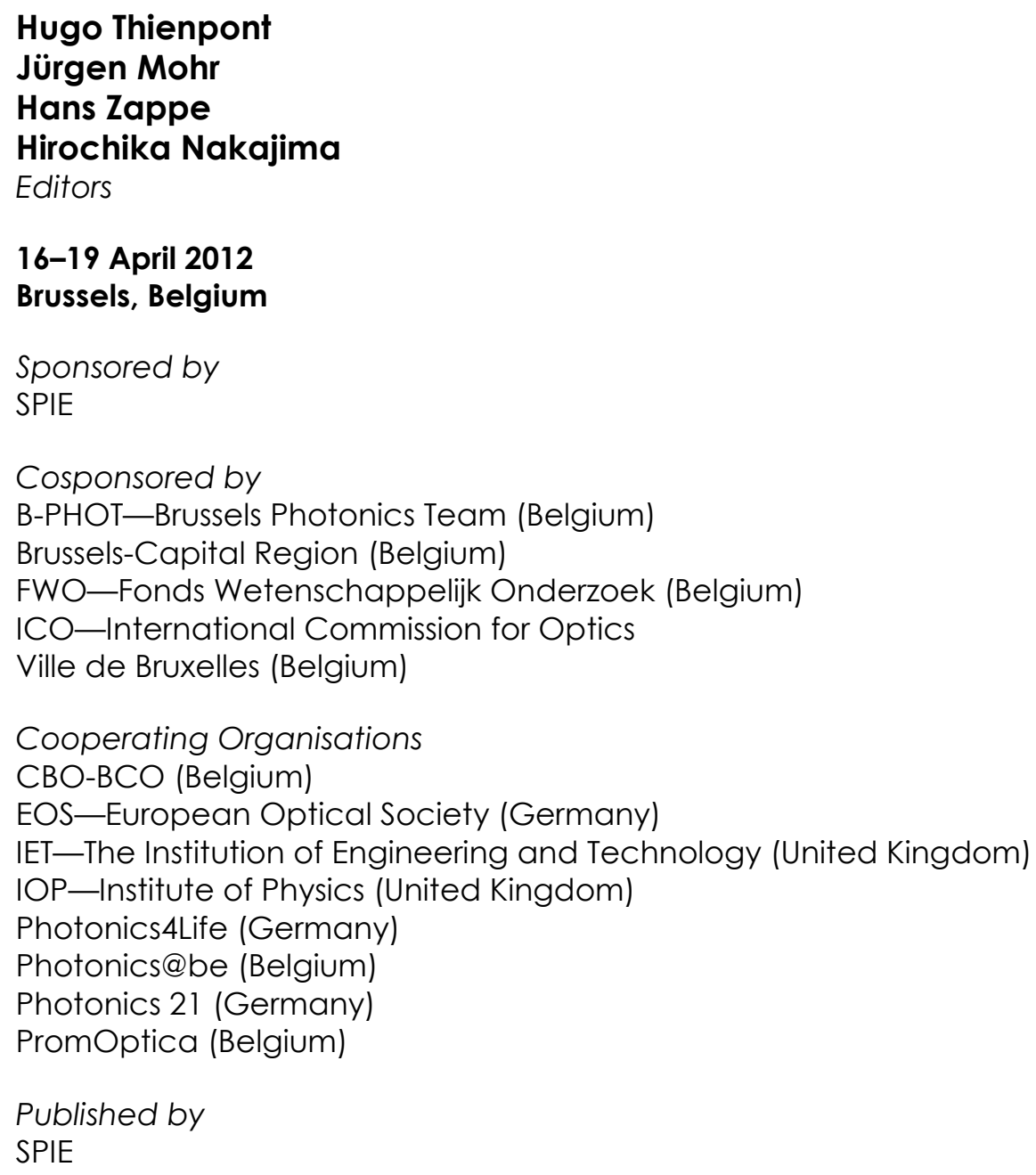


The papers included in this volume were part of the technical conference cited on the cover and title page. Papers were selected and subject to review by the editors and conference program committee. Some conference presentations may not be available for publication. The papers published in these proceedings reflect the work and thoughts of the authors and are published herein as submitted. The publisher is not responsible for the validity of the information or for any outcomes resulting from reliance thereon.

Please use the following format to cite material from this book:

Author(s), "Title of Paper," in Micro-Optics 2012, edited by Hugo Thienpont, Jürgen Mohr, Hans Zappe, Hirochika Nakajima, Proceedings of SPIE Vol. 8428 (SPIE, Bellingham, WA, 2012) Article CID Number.

ISSN 0277-786X

ISBN 9780819491206

Published by

SPIE

P.O. Box 10, Bellingham, Washington 98227-0010 USA

Telephone +1 3606763290 (Pacific Time) · Fax +1 3606471445

SPIE.org

Copyright (C) 2012, Society of Photo-Optical Instrumentation Engineers

Copying of material in this book for internal or personal use, or for the internal or personal use of specific clients, beyond the fair use provisions granted by the U.S. Copyright Law is authorized by SPIE subject to payment of copying fees. The Transactional Reporting Service base fee for this volume is $\$ 18.00$ per article (or portion thereof), which should be paid directly to the Copyright Clearance Center (CCC), 222 Rosewood Drive, Danvers, MA 01923. Payment may also be made electronically through CCC Online at copyright.com. Other copying for republication, resale, advertising or promotion, or any form of systematic or multiple reproduction of any material in this book is prohibited except with permission in writing from the publisher. The CCC fee code is 0277-786X/12/\$18.00.

Printed in the United States of America.

Publication of record for individual papers is online in the SPIE Digital Library.

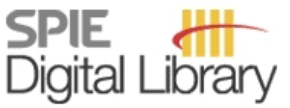

SPIEDigitalLibrary.org

Paper Numbering: Proceedings of SPIE follow an e-First publication model, with papers published first online and then in print and on CD-ROM. Papers are published as they are submitted and meet publication criteria. A unique, consistent, permanent citation identifier (CID) number is assigned to each article at the time of the first publication. Utilization of CIDs allows articles to be fully citable as soon as they are published online, and connects the same identifier to all online, print, and electronic versions of the publication. SPIE uses a six-digit CID article numbering system in which:

- The first four digits correspond to the SPIE volume number.

- The last two digits indicate publication order within the volume using a Base 36 numbering system employing both numerals and letters. These two-number sets start with $00,01,02,03,04$, $05,06,07,08,09,0 A, 0 B \ldots 0 Z$, followed by 10-1Z, 20-2Z, etc.

The CID number appears on each page of the manuscript. The complete citation is used on the first page, and an abbreviated version on subsequent pages. Numbers in the index correspond to the last two digits of the six-digit CID number. 


\section{Contents}

ix Conference Committee

\section{SESSION 1 NANOIMPRINT LITHOGRAPHY I}

842802 Pattern definition by nanoimprint (Invited Paper) [8428-01]

H.-C. Scheer, Bergische Univ. Wuppertal (Germany)

\section{SESSION 2 NANOIMPRINT LITHOGRAPHY II}

842807 Metallic colour filtering arrays manufactured by nanoimprint lithography (Invited Paper) [8428-06]

S. Landis, P. Brianceau, N. Chaix, Y. Désières, V. Reboud, M. Argoud, CEA-LETI, Minatec (France)

8428 OB Enhancement of extraction efficiency in nanoimprinted optical device structures (Invited Paper) [8428-10]

V. Reboud, Catalan Institute of Nanotechnology (Spain) and CEA-LETI, MINATEC (France); C. M. Sotomayor Torres, Catalan Institute of Nanotechnology (Spain), Catalan Institute for Research and Advanced Studies ICREA (Spain), and Univ. Autonoma de Barcelona (Spain)

\section{SESSION 3 GRATINGS AND HOLOGRAPHIC OPTICAL ELEMENTS}

8428 OC Volume Bragg gratings as ultra-narrow and multiband optical filters (Invited Paper) [8428-11]

A. L. Glebov, O. Mokhun, OptiGrate Corp. (United States); A. Rapaport, S. Vergnole, Horiba Scientific (France); V. Smirnov, L. B. Glebov, OptiGrate Corp. (United States)

8428 OD Holographic exposure of subwavelength circular gratings using a cone based interferogram [8428-12]

J. Sauvage-Vincent, Lab. Hubert Curien, CNRS (France) and Hologram Industries (France);

S. Tonchev, Y. Jourlin, O. Parriaux, Lab. Hubert Curien, CNRS (France)

8428 OE Optical diffraction into thick slab waveguides: a finite-beam RCWA approach to solve extremely asymmetrical scattering (EAS) in slanted holographic gratings [8428-13]

S. M. Pietralunga, Istituto di Fotonica e Nanotecnologie, CNR, Politecnico di Milano (Italy);

A. Geroldi, M. Serafini, Politecnico di Milano (Italy)

8428 OF Industrial fabrication of an optical security device for document protection using plasmon resonant transmission through a thin corrugated metallic film embedded on a plastic foil [8428-14]

J. Sauvage-Vincent, Hologram Industries (France) and Lab. Hubert Curien, CNRS (France);

Y. Jourlin, Lab. Hubert Curien, CNRS (France); S. Tonchev, Lab. Hubert Curien, CNRS (France) and Institute of Solid State Physics (Bulgaria); C. Veillas, C. Pedri, O. Parriaux, Lab. Hubert Curien, CNRS (France) 
$8428 \mathrm{OH}$ Interferometric inscription of volume Bragg gratings in a commercial high-refractive index glass (S-TIH53) by 400nm femtosecond (fs) laser pulses [8428-16]

T. Elsmann, A. Hartung, E. Lindner, M. Rothhardt, H. Bartelt, Institut für Photonische

Technologien e.V. (Germany)

\section{SESSION 4 FABRICATION AND PACKAGING OF MICRO-OPTICAL COMPONENTS}

8428 Ol Design and fabrication of advanced fiber alignment structures for field-installable fiber connectors (Invited Paper) [8428-17]

J. Van Erps, M. Vervaeke, A. Sánchez Martínez, Vrije Univ. Brussel (Belgium); S. Beri, TE Connectivity Ltd. (Belgium); C. Debaes, Vrije Univ. Brussel (Belgium); J. Watté, TE

Connectivity Ltd. (Belgium); H. Thienpont, Vrije Univ. Brussel (Belgium)

8428 0J Micromanipulators for a flexible automated assembly of micro optics [8428-18]

C. Brecher, N. Pyschny, S. Haag, V. Guerrero Lule, Fraunhofer-Institut für

Produktionstechnologie (Germany)

8428 OK Single-step direct laser fabrication of complex shaped microoptical components (Best Student Paper Award) [8428-19]

A. Žukauskas, K. K. Tikuišis, M. Ščiuka, A. Melninkaitis, R. Gadonas, Vilnius Univ. (Lithuania);

C. Reinhardt, Laser Zentrum Hannover e.V. (Germany); M. Malinauskas, Vilnius Univ.

(Lithuania)

8428 OL Micro-optical foundry: 3D lithography by freezing liquid instabilities at nanoscale (Invited Paper) [8428-20]

S. Grilli, Istituto Nazionale di Ottica (Italy); S. Coppola, Istituto Nazionale di Ottica (Italy) and Univ. degli Studi di Napoli Federico II (Italy); V. Vespini, F. Merola, A. Finizio, P. Ferraro, Istituto Nazionale di Ottica (Italy)

\section{SESSION 5 WAFER-LEVEL OPTICS AND MICROLENSES}

8428 ON Polymer tunable microlens arrays suitable for VCSEL beam control [8428-22]

B. Reig, V. Bardinal, T. Camps, Lab. d'Analyse et d'Architecture des Systèmes, CNRS (France) and Univ. de Toulouse (France); Y. G. Boucher, Univ. Européenne de Bretagne (France) and CNRS, FOTON, ENSSAT (France); C. Levallois, Univ. Européenne de Bretagne (France) and CNRS, FOTON, INSA (France); J. B. Doucet, D. Bourrier, E. Daran, J. Launay, Lab. d'Analyse et d'Architecture des Systèmes, CNRS (France) and Univ. de Toulouse (France)

842800 Fabrication of optical microlenses by a new inkjet printing technique based on pyro-electrohydrodynamic (PEHD) effect [8428-23]

S. Coppola, V. Vespini, Istituto Nazionale di Ottica (Italy); I. A. Grimaldi, F. Loffredo, F. Villani, ENEA (Italy); L. Miccio, S. Grilli, P. Ferraro, Istituto Nazionale di Ottica (Italy)

8428 OP Fabrication and test of polymeric microaxicons [8428-24]

F. Merola, Istituto Nazionale di Ottica (Italy); S. Coppola, Istituto Nazionale di Ottica (Italy) and Univ. degli Studi di Napoli (Italy); V. Vespini, S. Grilli, P. Ferraro, Istituto Nazionale di Ottica (Italy); D. Balduzzi, A. Galli, R. Puglisi, Istituto Sperimentale Italiano Lazzaro Spallanzani (Italy) 
$84280 Q \quad$ Large diameter multilevel graded nanostructured microlens [8428-25]

J. Nowosielski, R. Buczynski, Heriot-Watt Univ. (United Kingdom) and Univ. of Warsaw (Poland); A. J. Waddie, Heriot-Watt Univ. (United Kingdom); A. Filipkowski, Heriot-Watt Univ. (United Kingdom) and Institute of Electronic Materials Technology (Poland); D. Pysz, Institute of Electronic Materials Technology (Poland); A. McCarthy, Heriot-Watt Univ. (United Kingdom); R. Stepien, Institute of Electronic Materials Technology (Poland);

M. R. Taghizadeh, Heriot-Watt Univ. (United Kingdom)

\section{SESSION 6 MICRO-OPTICS STRUCTURES AND MATERIALS}

8428 OR Customised birefringence in nanostructured micro-optical devices (Invited Paper) [8428-26] A. J. Waddie, J. M. Nowosielski, A. Filipkowski, Heriot-Watt Univ. (United Kingdom); R. Stepien, D. Pysz, Institute of Electronic Materials Technology (Poland); R. Buczynski, Heriot-Watt Univ. (United Kingdom) and Univ. of Warsaw (Poland); M. R. Taghizadeh, Heriot-Watt Univ. (United Kingdom)

8428 OS Micromachining of optical fibers using selective etching of doped silica glass [8428-27] S. Pevec, E. Cibula, Univ. of Maribor (Slovenia); B. Lenardič, Optacore d.o.o (Slovenia); D. Đonlagić, Univ. of Maribor (Slovenia)

8428 OT Micro-optical elements and optical materials of certain spider webs [8428-28] D. M. Kane, N. Naidoo, D. J. Little, Macquarie Univ. (Australia)

$8428 \mathrm{OU}$ Focused ion beam sectioning of micro-optics as a tool for destructive testing for optical material [8428-29]

D. M. Kane, Macquarie Univ. (Australia); R. J. Chater, D. S. McPhail, Imperial College London (United Kingdom)

$8428 \mathrm{OV}$ Scalar diffraction theory for azimuthally structured Fresnel zone plate [8428-30]

T. Vierke, J. Jahns, FernUniv. in Hagen (Germany)

\section{SESSION 7 MICRO-OPTICS IN DISPLAY AND SPECTROSCOPY}

8428 0X Microstructured head-up display screen for automotive applications [8428-32]

M. K. Hedili, Koç Univ. (Turkey); M. O. Freeman, Microvision, Inc. (United States); H. Urey, Koç Univ. (Turkey)

8428 OY Single-shot-capable fast multichannel Fourier transform interferometer based on a microfabricated 3D multimirror array [8428-33]

H. O. Moser, Karlsruher Institut für Technologie (Germany) and National Univ. of Singapore

(Singapore); S. P. Heussler, S. M. P. Kalaiselvi, National Univ. of Singapore (Singapore)

$84280 Z$ Photonically enchanced polymer labs-on-a-chip [8428-34]

H. Ottevaere, J. Van Erps, M. Vervaeke, H. Thienpont, Vrije Univ. Brussel (Belgium) 
842810 3D tracking and phase-contrast imaging by twin-beams digital holographic microscope in microfluidics [8428-35]

L. Miccio, P. Memmolo, A. Finizio, M. Paturzo, F. Merola, S. Grilli, P. Ferraro, Istituto Nazionale di Ottica (Italy)

842812 Microoptical device for efficient read-out of active WGM resonators [8428-37]

T. Wienhold, M. Brammer, T. Grossmann, M. Schneider, H. Kalt, T. Mappes, Karlsruher Institut

für Technologie (Germany)

\section{SESSION 9 MICRO-OPTICS FOR SENSING AND MEMS}

842813 Optical characterization of a miniaturized large field-of-view motion sensor [8428-38]

E. Moens, H. Ottevaere, Y. Meuret, H. Thienpont, Vrije Univ. Brussel (Belgium)

842814 Long single-mode waveguides made by imprint patterning for optical interconnects and sensors [8428-39]

M. Karppinen, J. Hiltunen, A. Kokkonen, J. Petäjä, N. Masuda, M. Hiltunen, J. Tuominen,

P. Karioja, VTT Technical Research Ctr. of Finland (Finland)

842815 Novel gap alignment sensor for high-resolution proximity lithography [8428-40]

T. Harzendorf, Fraunhofer-Institut für Angewandte Optik und Feinmechanik (Germany);

L. Stuerzebecher, Friedrich-Schiller-Univ. Jena (Germany); U. D. Zeitner, Fraunhofer-Institut für Angewandte Optik und Feinmechanik (Germany) and Friedrich-Schiller-Univ. Jena (Germany)

842816 Micro-machined optical fibre cantilever as sensor elements [8428-41]

J. Li, F. Albri, R. R. J. Maier, W. N. MacPherson, D. P. Hand, Heriot-Watt Univ. (United Kingdom)

842817 Partially athermalized waveguide gratings [8428-70]

M. R. Saleem, Univ. of Eastern Finland (Finland) and National Univ. of Sciences and

Technology (Pakistan); S. Honkanen, J. Turunen, Univ. of Eastern Finland (Finland)

842818 3D rotating octagonal micromirror optical scanner: design, fabrication, and assembly [8428-43]

M. A. Basha, Univ. of Tabuk (Saudi Arabia)

\section{POSTER SESSION}

842819 Low f-number microlens array fabricated in thick resist [8428-44]

G. A. Cirino, A. N. Montagnoli, Univ. Federal de São Carlos (Brazil); P. Verdonck, IMEC (Belgium); L. G. Neto, Univ. de São Paulo (Brazil)

8428 1A Fabrication of near- or mid-infrared wire-grid polarizers with WSi wires [8428-45] I. Yamada, The Univ. of Shiga Prefecture (Japan); K. Fukumi, National Institute of Advanced Industrial Science and Technology (Japan); J. Nishii, Hokkaido Univ. (Japan); M. Saito, Ryukoku Univ. (Japan) 
8428 1B New industrial and innovative writing machine for the fabrication of sol-gel $\mathrm{TiO}_{2}$ based sub-micrometric period diffraction gratings [8428-46]

V. Gâté, Lab. Hubert Curien, CNRS, Univ. Jean Monnet (France) and Lab. des Matériaux et de Génie Physique, CNRS (France); G. Bernaud, C. Veillas, A. Cazier, Y. Jourlin, Lab. Hubert Curien, CNRS, Univ. Jean Monnet (France); M. Langlet, Lab. des Matériaux et de Génie Physique, CNRS (France); F. Vocanson, Lab. Hubert Curien, CNRS, Univ. Jean Monnet (France); P. Coudray, KLOÉ SA (France)

$84281 \mathrm{C}$ Highly efficient relief diffraction gratings inscribed in a chalcogenide bulk glass by a femtosecond laser [8428-47]

T. Kohoutek, Toyota Technological Institute (Japan); M. A. Hughes, Univ. of Surrey (United Kingdom); H. Kawashima, Toyota Technological Institute (Japan); M. Matsumoto, T. Misumi, Furukawa Denshi Co., Ltd. (Japan); T. Suzuki, Y. Ohishi, Toyota Technological Institute (Japan)

8428 1D Laser stabilized by acousto-optic cells for optoelectronic oscillators [8428-48] P. Salzenstein, FEMTO-ST, CNRS (France); A. S. Trushin, V. B. Voloshinov, Lomonosov Moscow State Univ. (Russian Federation)

8428 IE Design of optical interconnects inspired in multi-aperture optics based in compound insect eyes [8428-49]

A. Garza-Rivera, C.-G. Trevino-Palacios, F. J. Renero-Carrillo, Instituto Nacional de Astrofísica, Óptica y Electrónica (Mexico)

$8428 \mathrm{IF}$ Design of a novel pump for bio-applications [8428-50]

S.-L. Chang, K.-C. Chiu, Industrial Technology Research Institute (Taiwan); F.-Y. Hsu, National United Univ. (Taiwan); J.-K. Chen, National Taipei Univ. of Technology (Taiwan)

$84281 G \quad$ Fabrication of $100 \%$ fill factor arrays of microlenses from silicon molds [8428-51] M. Baranski, N. Passilly, J. Albero, C. Gorecki, FEMTO-ST, CNRS (France)

$8428 \mathrm{1H}$ Design and fabrication of micro-windmill for fluidic media based on SU-8 for using flow-meter [8428-52]

I. Sabri Alirezaei, M. Ghaderi, M. Khalafi, H. Latifi, Shahid Beheshti Univ. (Iran, Islamic Republic of)

842811 Design, fabrication, characterization of a polymeric nano-precision micro z-stage [8428-53] M. Ghaderi, Delft Univ. of Technology (Netherlands); F. Beygi Azar Aghbolagh, M. Taghavi, I. Sabri, M. Sadegh, H. Latifi, Shahid Beheshti Univ. (Iran, Islamic Republic of)

8428 IL Talbot-carpets of periodic and quasi-periodic close-packed 2D mask structures calculated by a modified chirp-z-algorithm [8428-55]

J.S. Maaß, Carl Zeiss Jena GmbH (Germany); O. Sandfuchs, Univ. of Applied Sciences Hamm-Lippstadt (Germany); A. Gatto, Carl Zeiss Jena GmbH (Germany); D. Thomae, R. Brunner, Univ. of Applied Sciences, Jena (Germany)

$84281 \mathrm{M}$ The refractive index measurement technique based on the defocus correction method in full-field optical coherence tomography [8428-57]

G. Min, J. W. Kim, B.-H. Lee, Gwangju Institute of Science and Technology (Korea, Republic of) 
$842810 \quad$ Fabry-Perot tunable infrared filter based on structured reflectors [8428-59]

S. Kurth, Fraunhofer ENAS (Germany); K. Hiller, Chemnitz Univ. of Tech. (Germany);

N. Neumann, M. Seifert, M. Ebermann, InfraTec GmbH (Germany); H. Specht, M. Meinig,

T. Gessner, Fraunhofer ENAS (Germany)

8428 IP Development of glass microoptics for MidIR with hot embossing technology [8428-60] I. Kujawa, R. Stepien, Institute of Electronic Materials Technology (Poland); A. J. Waddie, Heriot-Watt Univ. (United Kingdom); G. Skrabalak, Institute of Advanced Manufacturing Technology (Poland); M. R. Taghizadeh, Heriot-Watt Univ. (United Kingdom); R. Buczynski, Institute of Electronic Materials Technology (Poland) and Univ. of Warsaw (Poland)

8428 IR Diffractive optics development with stack-and-draw technique [8428-62]

J. M. Nowosielski, Heriot-Watt Univ. (United Kingdom) and Univ. of Warsaw (Poland); A. Filipkowski, Heriot-Watt Univ. (United Kingdom) and Institute of Electronic Materials Technology (Poland); A. J. Waddie, Heriot-Watt Univ. (United Kingdom); I. Kujawa, R. Stepien, Institute of Electronic Materials Technology (Poland); R. Buczynski, Univ. of Warsaw (Poland) and Institute of Electronic Materials Technology (Poland);

M. R. Taghizadeh, Heriot-Watt Univ. (United Kingdom)

$84281 \mathrm{~T}$ Development of light-scattering thermal cross-linking package film based on self-assembly for liquid crystal display using light emitting diode [8428-64]

S. Takei, Toyama Prefectural Univ. (Japan) and Osaka Univ. (Japan); K. Mochizuki, N. Kubo, Toyama Prefectural Univ. (Japan); Y. Yokoyama, Toyama Industrial Technology Ctr. (Japan)

$84281 \mathrm{U}$ Development of plant-based resist material derived from biomass on hardmask layer in ultraviolet curing nanoimprint lithography [8428-65]

S. Takei, Toyama Prefectural Univ. (Japan) and Osaka Univ. (Japan)

$84281 \mathrm{~V}$ Development of water-developable resist material derived from biomass in EB lithography [8428-66]

S. Takei, Toyama Prefectural Univ. (Japan) and Osaka Univ. (Japan); A. Oshima, Osaka Univ. (Japan); T. Wakabayashi, A. Sekiguchi, Toyama Prefectural Univ. (Japan); T. Kozawa,

S. Tagawa, Osaka Univ. (Japan) and Japan Science and Technology Agency (Japan)

8428 IW Physical and mechanical properties of a TIR-based liquid micro deformable mirror [8428-67] E. S. ten Have, Delft Univ. of Technology (Netherlands); G. Vdovin, Delft Univ. of Technology (Netherlands) and Flexible Optical B.V. (Netherlands)

$84281 \mathrm{X} \quad$ Giant increase of photorefractive effect in lithium niobate: a novel approach [8428-68] M. Bazzan, M. Michieletto, L. Bacci, N. Argiolas, A. Zaltron, M. V. Ciampolillo, G. Pozza, C. Sada, Univ. degli Studi di Padova (Italy)

$84281 \mathrm{Y}$ Investigation on 2D disks and stadiums micro-resonators structures based on UV210 polymer [8428-71]

D. Pluchon, N. Huby, IPR, CNRS, Univ. de Rennes 1 (France); H. Lhermite, IETR, CNRS, Univ. de Rennes 1 (France); D. Duval, Univ. Autònoma de Barcelona (Spain); B. Bêche, IPR, CNRS, Univ. de Rennes 1 (France) and Institut universitaire de France (France) 
$84281 Z$ Controlled USP laser ablation strategies for shaping optics [8428-72]

C. Schindler, Fachhochschule Jena (Germany); J. Giesecke, Günter-Köhler-Institut für Fügetechnik und Werkstoffprüfung GmbH (Germany); J. Bliedtner, Fachhochschule Jena (Germany); H. Mueller, S. Waechter, Günter-Köhler-Institut für Fügetechnik und Werkstoffprüfung GmbH (Germany); V. Giggel, Carl Zeiss Jena GmbH (Germany)

Author Index 
Downloaded From: https://www.spiedigitallibrary.org/conference-proceedings-of-spie on 26 Apr 2023

Terms of Use: https://www.spiedigitallibrary.org/terms-of-use 


\title{
Conference Committee
}

\author{
Symposium Chairs
}

Francis Berghmans, Vrije Universiteit Brussel (Belgium)

Ronan Burgess, European Commission (Belgium)

Jürgen Popp, Institut für Photonische Technologien e.V. (Germany)

Peter Hartmann, SCHOTT AG (Germany)

Honorary Symposium Chair

Hugo Thienpont, Vrije Universiteit Brussel (Belgium)

\section{Conference Chairs}

Hugo Thienpont, Vrije Universiteit Brussel (Belgium)

Jürgen Mohr, Karlsruher Institut für Technologie (Germany)

Hans Zappe, Universität Freiburg (Germany)

Hirochika Nakajima, Waseda University (Japan)

\section{Programme Committee}

Véronique Bardinal, Laboratoroire d'Analyse et d'Architecture des Systèmes (France)

Francis Berghmans, Vrije Universiteit Brussel (Belgium)

Pierre H. Chavel, Institut d'Optique Graduate School (France)

Pietro Ferraro, Istituto Nazionale di Ottica (Italy)

Dietmar Fey, Friedrich-Alexander-Universität Erlangen-Nürnberg (Germany)

Michael A. Fiddy, The University of North Carolina at Charlotte (United States)

Eric Fogarassy, Institut d'Électronique du Solide et des Systèmes (France)

Alexei L. Glebov, OptiGrate Corporation (United States)

Jürgen Jahns, FernUniversität in Hagen (Germany)

Pentti Karioja, VTT Technical Research Center of Finland (Finland)

Bernard Kress, USI Photonics Inc. (United States)

El-Hang Lee, Inha University (Korea, Republic of)

Marc J. Madou, University of California, Irvine (United States)

Olivier M. Parriaux, Université Jean Monnet Saint-Etienne (France)

Oltmann Riemer, Universität Bremen (Germany)

Clivia M. Sotomayor Torres, Institut Català de Nanotecnologia (Spain)

Ion G. Stiharu, Concordia University (Canada)

Peter Van Daele, Universiteit Gent (Belgium)

Henne van Heeren, Enabling M3 (Netherlands)

Geert Van Steenberge, Universiteit Gent (Belgium) 
Session Chairs

1 Nanoimprint Lithography I

Clivia M. Sotomayor Torres, Institut Català de Nanotecnologia (Spain)

2 Nanoimprint Lithography II

Jouni Ahopelto, NaPa (Finland)

3 Gratings and Holographic Optical Elements

Olivier M. Parriaux, Laboratoroire Hubert Curien (France)

$4 \quad$ Fabrication and Packaging of Micro-Optical Components

Peter Van Daele, Universiteit Gent (Belgium)

$5 \quad$ Wafer-level Optics and Microlenses

Juergen Mohr, Karlsruher Institut für Technologie (Germany)

6 Micro-optics Structures and Materials

Hans Zappe, Albert-Ludwigs-Universität Freiburg (Germany)

$7 \quad$ Micro-optics in Display and Spectroscopy

Alexei L. Glebov, OptiGrate Corporation (United States)

8 Micro-optics Lab-on-a-chip

Bernard C. Kress, USI Photonics Inc. (United States)

9 Micro-optics for Sensing and MEMS

Olivier M. Parriaux, Laboratoroire Hubert Curien (France) 